\title{
Altered Superficial White Matter on Tractography MRI in Alzheimer's Disease
}

\author{
William Reginold ${ }^{a}, b \quad$ Angela C. Luedke ${ }^{b}$ Justine Itorralba ${ }^{b}$ \\ Juan Fernandez-Ruiz ${ }^{d} \quad$ Omar Islam $^{c}$ Angeles Garciab \\ a Department of Medical Imaging, University of Toronto, Toronto, Ont., b Memory \\ Clinics, Division of Geriatric Medicine, Department of Medicine, Queen's University, and \\ 'Department of Diagnostic Radiology, Kingston General Hospital, Queen's University, \\ Kingston, Ont., Canada; ${ }^{\mathrm{d}}$ Facultad de Medicina, Universidad Nacional Autonoma de Mexico, \\ Coyoacán, Mexico
}

\author{
Key Words \\ Cognitive function · Diffusion tensor imaging $\cdot$ Tractography
}

\section{Abstract}

Background/Aims: Superficial white matter provides extensive cortico-cortical connections. This tractography study aimed to assess the diffusion characteristics of superficial white matter tracts in Alzheimer's disease. Methods: Diffusion tensor 3T magnetic resonance imaging scans were acquired in 24 controls and 16 participants with Alzheimer's disease. Neuropsychological test scores were available in some participants. Tractography was performed by the Fiber Assignment by Continuous Tracking (FACT) method. The superficial white matter was manually segmented and divided into frontal, parietal, temporal and occipital lobes. The mean diffusivity (MD), radial diffusivity (RD), axial diffusivity (AxD) and fractional anisotropy (FA) of these tracts were compared between controls and participants with Alzheimer's disease and correlated with available cognitive tests while adjusting for age and white matter hyperintensity volume. Results: Alzheimer's disease was associated with increased MD ( $p=$ $0.0011)$, increased RD ( $p=0.0019)$ and increased $\operatorname{AxD}(p=0.0017)$ in temporal superficial white matter. In controls, superficial white matter was associated with the performance on the Montreal Cognitive Assessment, Stroop and Trail Making Test B tests, whereas in Alzheimer's disease patients, it was not associated with the performance on cognitive tests. Conclusion: Temporal lobe superficial white matter appears to be disrupted in Alzheimer's disease. 
Reginold et al.: Altered Superficial White Matter on Tractography MRI in Alzheimer's Disease

\section{Introduction}

Disruption of cerebral white matter is seen in Alzheimer's disease (AD) [1]. According to the retrogenesis model of $\mathrm{AD}$, the white matter tracts that are the last to myelinate are the first to degenerate [2]. Superficial white matter (SWM) is located beneath the infragranular layer of the cerebral cortex and these tracts are some of the last fibers to myelinate, often occurring in the fourth decade of life [3]. Therefore, SWM represents a population of tracts that may be most vulnerable to AD. SWM consists of U fibers of Meynert, crown fibers and merging deep white matter fibers [4]. U fibers originate from layers III and V of the cortex, exit and follow concave surfaces of a sulcus and re-enter the cortex at distances up to $3 \mathrm{~cm}$. Crown fibers run parallel to the convex surface of gyri. Merging fibers originate within the deep white matter, cross other tracts in SWM and extend towards the cortex. Perhaps due to the complex architecture and significant inter-subject variability of SWM, there have been limited studies of its involvement in AD and cognitive dysfunction. A magnetization transfer ratio magnetic resonance imaging (MRI) study found evidence of demyelination of SWM in $\mathrm{AD}$ and an association with lower Mini-Mental State Examination scores [3]. A diffusion tensor MRI study found decreased white matter integrity of SWM in AD, particularly in the frontal and temporal lobes and associations with lower Mini-Mental State Examination scores [5]. Additional imaging studies are needed to characterize the alterations of SWM in AD and its cognitive correlates.

Tractography is an application of diffusion tensor MRI that reconstructs white matter tracts based on restricted diffusion of water molecules along myelinated axons [6]. Disruption of tracts can be detected as increased mean diffusivity (MD, degree of water diffusion), increased axial diffusivity (AxD, largest eigenvalue), increased radial diffusivity ( $R D$, average of the two smaller eigenvalues) or decreased fractional anisotropy (FA, directional diffusion). A tractography study in healthy older adults demonstrated an association between lower SWM FA and lower scores on cognitive testing [7]. Earlier tractography studies in AD assessed lobar white matter that likely consisted of both SWM and deep white matter, but none assessed only SWM [8-14]. The aim of this study was to use tractography to characterize alterations in SWM associated with $\mathrm{AD}$. We used this method to reconstruct each participant's unique network of SWM tracts. Since AD has differential effects across cerebral lobes, we separately assessed frontal, occipital, parietal and temporal SWM. In each lobe, we compared the diffusion characteristics (MD, AxD, RD and FA) of SWM tracts between healthy older adults and persons with $\mathrm{AD}$ and correlated it with the performance on neuropsychological testing. We hypothesized that AD would be associated with worse SWM diffusion characteristics (increased MD/AxD/RD and decreased FA). We also hypothesized that worse SWM diffusion characteristics would be associated with decreased cognitive function.

\section{Methods}

\section{Participants and Clinical Assessment}

The study was approved by the Queen's University Research Ethics Board. Prior to entering the study, all participants provided written informed consent. The neuropsychological battery consisted of the following cognitive tests: global cognitive function (Montreal Cognitive Assessment, MoCA [15] and Mini-Mental State Examination [16]), selective attention (Stroop test [17]), processing speed (Trail Making Test B, Trail [18]), focused attention (Wechsler Memory Scale-III Longest Span forward [19]) and working memory (Wechsler Memory Scale-III Longest Span backward and Letter Number Sequencing [19]). AD was diag- 
Reginold et al.: Altered Superficial White Matter on Tractography MRI in Alzheimer's Disease

nosed following the NINCDS-ADRDA criteria [20]. Participants with mild cognitive impairment were excluded from this study. Participants with AD were within the mild stages of the disease, as measured by a MoCA score of $\geq 18 / 30$. Exclusion criteria were the presence of metallic objects, devices or conditions unsafe for MRI. The cognitive testing and MRI session were conducted within 2 weeks of each other. Twenty-four cognitive normal controls and 16 participants with AD were included in the study. The demographic characteristics of the participants are shown in table 1.

\section{Magnetic Resonance Imaging}

Brain imaging was acquired in a single session on a 3 Tesla Siemens Magnetom Trio MRI system (Siemens Medical Systems, Erlangen, Germany) with the use of a 12-channel head coil. An anatomical scan was acquired with a sagittal T1-weighted 3-dimensional magnetization prepared rapid gradient echo sequence [field of view (FoV) $256 \mathrm{~mm}$, spatial resolution $1 \times 1$ $\times 1 \mathrm{~mm}^{3}$, repetition time (TR) $1,760 \mathrm{~ms}$, echo time (TE) $2.2 \mathrm{~ms}$, flip angle $9^{\circ}$, number of slices 176]. An axial T2-weighted 2-dimensional fluid-attenuated inversion recovery sequence (FLAIR) interleaved scan was acquired (FoV $250 \mathrm{~mm}$, voxel size $1 \times 1 \times 3 \mathrm{~mm}^{3}$, TR 9,000 ms, TE $79 \mathrm{~ms}$, flip angle $180^{\circ}$, number of slices 40). Diffusion tensor imaging (DTI) data were acquired in 30 directions using a single-shot echo planar imaging sequence with 31 volumes of 60 axial slices (b-value $1=0 \mathrm{~s} / \mathrm{mm}^{2}$, and b-value $2=1,000 \mathrm{~s} / \mathrm{mm}^{2}$ ), slice thickness $2 \mathrm{~mm}$, $\mathrm{TR} / \mathrm{TE}=7,800 / 95 \mathrm{~ms}$, FoV $256 \times 256 \mathrm{~mm}^{2}$ and an acquisition matrix of $128 \times 128$, resulting in a resolution of $2 \times 2 \times 2 \mathrm{~mm}^{3}$.

\section{Image Analysis}

Diffusion-weighted images were processed by a method described in earlier studies [21-23]. Diffusion-weighted images were corrected for eddy current distortions using FSL [24]. There was no correction for head motion. DTI reconstruction was completed with Diffusion Toolkit 0.5 (Ruopeng Wang, Van J. Wedeen, Martinos Center for Biomedical Imaging, Massachusetts General Hospital, www.trackvis.org). Tracts were created in the Diffusion Toolkit by the Fiber Assignment by Continuous Tracking (FACT) method with an angle threshold of $35^{\circ}$ [24]. The participant's T2 FLAIR MRI was registered by the affine method using 12 degrees of freedom to the diffusion-weighted image map with Slicer 3D 4.1 (www.slicer.org). Tractography data and T2 FLAIR were analyzed in TrackVis (www.trackvis.org). White matter hyperintensities (WMH) were manually segmented on axial T2 FLAIR as described in an earlier study [22]. SWM was defined as tracts originating within $5 \mathrm{~mm}$ of the cortical surface, and it was manually segmented into frontal, occipital, parietal and temporal lobe SWM based on anatomical landmarks [landmarks are described in ref. 25]. Within each lobe, region of interests (ROIs) were outlined paralleling the cortex within $5 \mathrm{~mm}$ of the cortical surface. ROIs were manually traced for each lobe, slice by slice, on the 40 axial T2 FLAIR slices for each patient using the TrackVis mouse-controlled interface. Frontal, occipital, parietal and temporal lobe SWM was segmented by selecting tracts that crossed through the respective lobar ROIs. The area of ROI as well as FA, MD, AxD and RD of the lobar SWM were measured. Examples of segmented SWM tracts are shown in figure 1.

\section{Statistical Analysis}

Statistical analyses were conducted using Statplus. The FA, MD, AxD and RD of segmented SWM tracts were compared between controls and participants with AD while adjusting for the effects of age and the WMH volume using multiple linear regressions. Cognitive scores were not available in all participants, so participants were included in the analysis only when their scores were available (sample sizes are presented in tables 3 and 4). We presented 

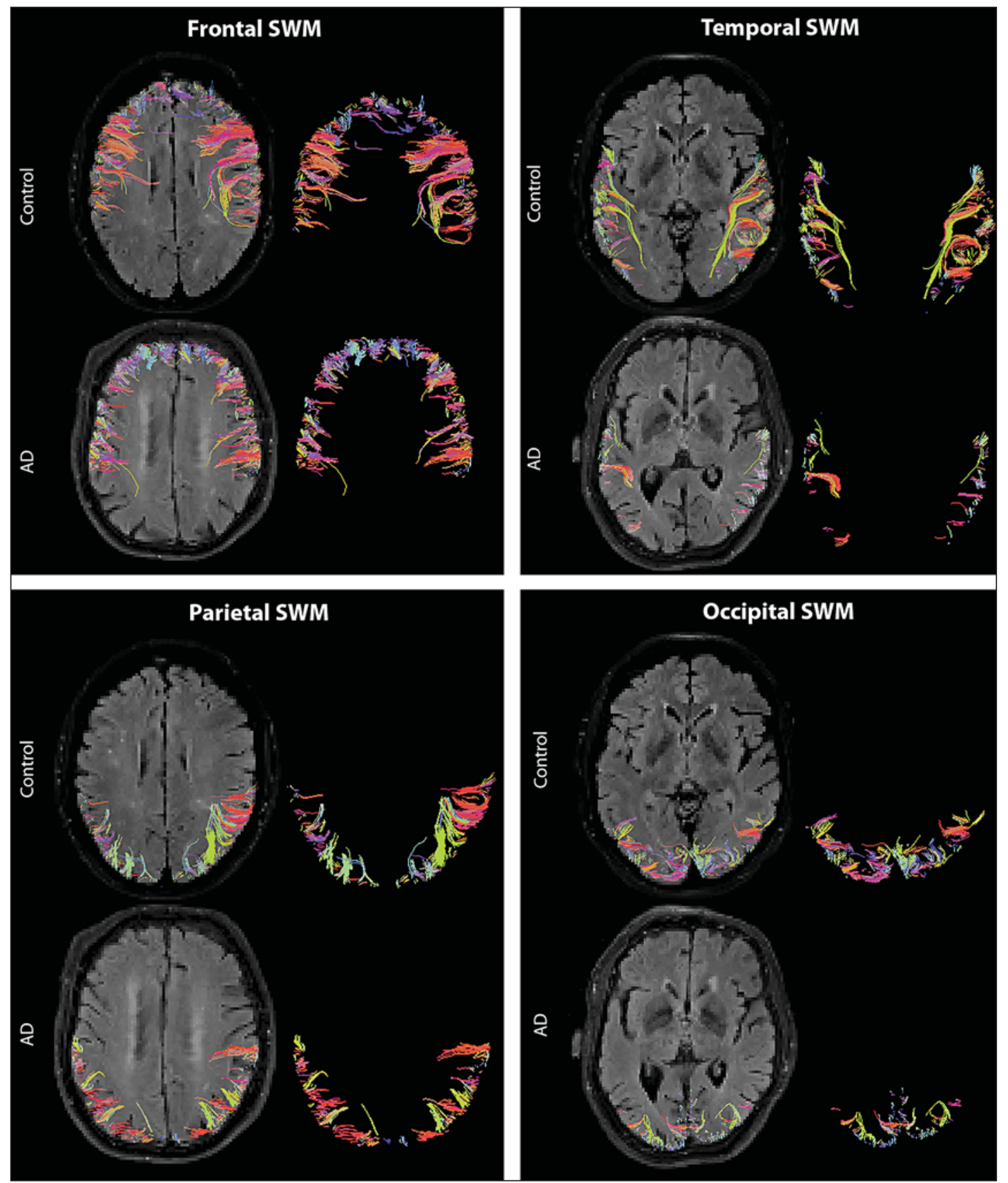

Fig. 1. Example of SWM tracts generated by tractography in axial sections in a participant with AD and a control. Examples of frontal, temporal, parietal and occipital SWM tracts in axial sections in each participant are shown overlying the corresponding axial T2 FLAIR image.

unstandardized regression coefficients for the associations between cognitive test scores with tracts $\mathrm{MD}, \mathrm{AxD}, \mathrm{RD}$ and $\mathrm{FA}$ and number using multiple linear regressions, adjusting for the effects of age and WMH volume. To account for comparison of the 4 SWM lobes, a Bonferroni correction was used, and $p$ values $<0.0125$ were considered statistically significant. 
Reginold et al.: Altered Superficial White Matter on Tractography MRI in Alzheimer's Disease

Table 1. Demographic and clinical data for cognitively normal controls and participants with AD

\begin{tabular}{lccc}
\hline & Controls & AD patients & p value \\
\hline Subjects, $\mathrm{n}$ & 24 & 16 & - \\
Age, years & $69.9 \pm 8.98$ & $73.7 \pm 7.14$ & 0.164 \\
M/F, n & $9 / 15$ & $7 / 9$ & 0.750 \\
WMH volume, voxels & $1,060 \pm 2,880$ & $3,600 \pm 6,530$ & 0.1005 \\
MoCA score (max. 30) & $27.9 \pm 1.67$ & $22.1 \pm 2.11$ & $<0.0001$ \\
MMSE score & $29.0 \pm 1.23$ & $24.4 \pm 0.97$ & $<0.0001$ \\
Stroop score (max. 112) & $92.5 \pm 14.7$ & $53.7 \pm 17.0$ & $<0.0001$ \\
Trail B, s & $95.6 \pm 35.3$ & $197 \pm 108$ & $<0.0001$ \\
Span forward score & $8.50 \pm 1.19$ & $6.60 \pm 1.43$ & $<0.0001$ \\
Span backward score & $7.85 \pm 1.27$ & $6.20 \pm 1.62$ & $<0.0001$ \\
Letter Number Sequencing score & $11.1 \pm 2.21$ & $7.00 \pm 3.20$ & $<0.0001$ \\
\hline
\end{tabular}

Values are means \pm standard deviations unless otherwise stated. MMSE = Mini-Mental State Examination; Span forward = Wechsler Memory Scale-III Longest span forward; Span backward = Wechsler Memory Scale-III Longest span backward.

\section{Results}

The demographic and clinical characteristics of participants are presented in table 1. There was a trend for increased age and increased WMH volume in participants with AD compared to controls; however, it was not statistically significant (table 1). As expected, participants with AD had statistically significantly lower scores on all cognitive tests compared to controls (table 1; all tests $\mathrm{p}<0.001$ ).

Compared to controls, the area of SWM was smaller in participants with AD (frontal SWM: $5,576 \pm 4,556$ vs. $1,119 \pm 517, \mathrm{p}=0.00073$; occipital SWM: $1,627 \pm 1,211$ vs. $316 \pm 155$, $\mathrm{p}=0.00025$; parietal SWM: $3,652 \pm 2,747$ vs. $687 \pm 161, \mathrm{p}=0.00015$; temporal SWM: 2,083 $\pm 1,706$ vs. $391 \pm 227, p=0.00029$ ). Compared to controls, in participants with AD, there was increased MD (percentage difference 12\%), increased $\mathrm{AxD}(9 \%)$ and increased RD in temporal SWM (14\%; table 2). There were no statistically significant differences in the FA of SWM between controls and participants with AD (table 2).

Among controls, in frontal SWM, increased RD and decreased FA were associated with worse MoCA scores (table 3). In occipital SWM, increased RD was associated with worse MoCA and Trail scores, and decreased FA was associated with worse Trail scores. In temporal SWM, decreased FA was associated with worse MoCA scores, and increased MD and RD were associated with worse Stroop scores. In participants with AD, the FA, MD, RD and AxD of SWM was not associated with the performance on the cognitive tests (table 4).

\section{Discussion}

This is the first tractography study to detect alterations in the SWM in AD. Compared to controls, AD was associated with worse SWM diffusion characteristics. The retrogenesis hypothesis predicted that, as late myelinating fibers, SWM would be vulnerable to degeneration in $\mathrm{AD}$ [2]. In support of the retrogenesis hypothesis, we detected disruption of SWM in AD. Our results are in agreement with an earlier magnetization transfer ratio imaging study and the DTI study that found evidence for disruption of SWM in AD $[3,5]$. 
Table 2. Comparison of tract FA, MD, RD and AxD between controls and participants with $\mathrm{AD}$ adjusting for age and $\mathrm{WMH}$ volume

\begin{tabular}{lccll}
\hline & Controls & AD & $\beta$ & \multicolumn{1}{l}{$\begin{array}{l}\text { Regression } \\
\text { p value }\end{array}$} \\
\hline$M D$ & & & & \\
Frontal & $0.001116 \pm 0.000201$ & $0.001219 \pm 0.000171$ & 0.00006 & 0.34618 \\
Occipital & $0.000880 \pm 0.000076$ & $0.000959 \pm 0.000099$ & 0.00007 & 0.0238 \\
Parietal & $0.00116 \pm 0.00026$ & $0.00132 \pm 0.00028$ & 0.00014 & 0.12998 \\
Temporal & $0.000863 \pm 0.000052$ & $0.000968 \pm 0.000108$ & 0.00009 & 0.00107 \\
\hline$R D$ & & & & \\
Frontal & $0.000999 \pm 0.000205$ & $0.001107 \pm 0.000171$ & 0.00006 & 0.32062 \\
Occipital & $0.000774 \pm 0.000084$ & $0.000856 \pm 0.000110$ & 0.00007 & 0.03286 \\
Parietal & $0.00104 \pm 0.00026$ & $0.00121 \pm 0.00027$ & 0.00014 & 0.12691 \\
Temporal & $0.000754 \pm 0.000058$ & $0.000862 \pm 0.000114$ & 0.00009 & 0.00185 \\
\hline AxD & & & & \\
Frontal & $0.001342 \pm 0.000196$ & $0.001433 \pm 0.000172$ & 0.00005 & 0.39726 \\
Occipital & $0.001082 \pm 0.000077$ & $0.001156 \pm 0.000090$ & 0.00006 & 0.02923 \\
Parietal & $0.00139 \pm 0.00026$ & $0.00154 \pm 0.00032$ & 0.00013 & 0.1987 \\
Temporal & $0.001076 \pm 0.000051$ & $0.001168 \pm 0.000097$ & 0.00008 & 0.00173 \\
\hline FA & & & & \\
Frontal & $0.270 \pm 0.0415$ & $0.241 \pm 0.029$ & -0.01606 & 0.14878 \\
Occipital & $0.257 \pm 0.0542$ & $0.238 \pm 0.053$ & 0.00007 & 0.0238 \\
Parietal & $0.251 \pm 0.044$ & $0.219 \pm 0.026$ & -0.02462 & 0.07023 \\
Temporal & $0.269 \pm 0.034$ & $0.241 \pm 0.0351$ & -0.02038 & 0.08202 \\
\hline & & & & \\
\hline
\end{tabular}

Values are means \pm standard deviations unless otherwise stated. $\beta$ represents the nonstandardized regression coefficient.

The pattern of SWM tract changes observed in this study was similar to earlier reported patterns of cortical neurodegeneration in AD. Based on the Braak staging system, neurofibrillary degeneration develops in the temporal lobe and spreads to the rest of the neocortex in later stages of the disease [26]. Consistent with its involvement in early stages of AD, in this study, we found the largest diffusion abnormalities in the temporal lobe. The large increases in $\mathrm{MD}, \mathrm{AxD}$ and RD within the temporal SWM ranged from 9 to $14 \%$ and are consistent with the $10-25 \%$ increase reported by an earlier DTI study [5]. It is believed that increased MD represents tissue atrophy, increased $\mathrm{AxD}$ represents Wallerian degeneration, and increased RD reflects myelin disruption [5]. Based on these associations, our study suggests that in AD, there is prominent tissue atrophy, Wallerian degeneration and myelin disruption in temporal SWM. Consistent with earlier studies, there were greater differences in MD, AxD and RD than in FA between controls and participants with early-stage $\mathrm{AD}[5,27]$. FA is related to the ratio of $\mathrm{AxD}$ and RD [27]. In this study, both AxD and RD were increased in participants with AD, which may have attenuated changes in FA in AD.

We detected novel associations between SWM and cognitive function. SWM had different associations with cognitive function based on its location. In healthy older adults, decreased global cognitive function was associated with worse diffusion characteristics of SWM in several lobes. Decreased performance on the MoCA was associated with increased RD/decreased FA in frontal SWM, increased RD in occipital SWM and decreased FA in temporal SWM. Decreased cognitive function in specific cognitive domains was associated with worse diffusion characteristics of SWM in specific lobes. Decreased selective attention assessed by the Stroop test 
Table 3. Regression coefficients of the correlation between white matter tract FA, MD, RD and AxD with cognitive test scores among controls

\begin{tabular}{|c|c|c|c|c|c|c|c|c|c|c|c|c|c|c|}
\hline & \multicolumn{2}{|c|}{$\operatorname{MoCA}(\mathrm{n}=24)$} & \multicolumn{2}{|c|}{ MMSE $(\mathrm{n}=20)$} & \multicolumn{2}{|c|}{ Stroop $(\mathrm{n}=22)$} & \multicolumn{2}{|c|}{ Trail $(\mathrm{n}=21)$} & \multicolumn{2}{|c|}{$\begin{array}{l}\text { Span forward } \\
(\mathrm{n}=18)\end{array}$} & \multicolumn{2}{|c|}{$\begin{array}{l}\text { Span } \\
\text { backward } \\
(\mathrm{n}=18)\end{array}$} & \multicolumn{2}{|c|}{$\begin{array}{l}\text { Letter } \\
\text { Number } \\
\text { Sequencing } \\
(\mathrm{n}=18)\end{array}$} \\
\hline & $\beta$ & $\mathrm{p}$ & $\beta$ & $\mathrm{p}$ & $\beta$ & $\mathrm{p}$ & $\beta$ & $\mathrm{p}$ & $\beta$ & $\mathrm{p}$ & $\beta$ & $\mathrm{p}$ & $\beta$ & $\mathrm{p}$ \\
\hline \multicolumn{15}{|l|}{$M D$} \\
\hline Frontal & $-3,770$ & 0.017 & 260 & 0.883 & $-41,500$ & 0.077 & 40,200 & 0.484 & $-3,170$ & 0.132 & $-1,060$ & 0.527 & -64.1 & 0.986 \\
\hline Occipital & $-9,880$ & 0.014 & 625 & 0.863 & $-92,700$ & 0.022 & 234,000 & 0.018 & $-7,220$ & 0.052 & $-1,360$ & 0.656 & $-8,430$ & 0.175 \\
\hline Parietal & $-2,360$ & 0.066 & -991 & 0.585 & $-30,000$ & 0.201 & 25,600 & 0.645 & $-4,830$ & 0.021 & $-1,350$ & 0.438 & 5,730 & 0.106 \\
\hline Temporal & $-12,000$ & 0.048 & 1,980 & 0.726 & $-181,000$ & 0.004 & 161,000 & 0.342 & $-6,580$ & 0.305 & 3,440 & 0.489 & 1,740 & 0.869 \\
\hline \multicolumn{15}{|l|}{$R D$} \\
\hline Frontal & $-3,890$ & 0.011 & 217 & 0.902 & $-40,450$ & 0.081 & 48,900 & 0.385 & $-3,400$ & 0.104 & $-1,100$ & 0.513 & -183 & 0.959 \\
\hline Occipital & $-9,460$ & 0.008 & $-1,340$ & 0.694 & $-80,800$ & 0.027 & 233,000 & 0.005 & $-6,680$ & 0.057 & -357 & 0.901 & $-8,390$ & 0.150 \\
\hline Parietal & $-2,400$ & 0.059 & -780 & 0.678 & $-30,600$ & 0.195 & 31,100 & 0.581 & $-5,040$ & 0.018 & $-1,420$ & 0.428 & 5,640 & 0.122 \\
\hline Temporal & $-13,200$ & 0.013 & 1,250 & 0.811 & $-153,000$ & 0.009 & 200,000 & 0.182 & $-7,000$ & 0.224 & 3,200 & 0.476 & 2,840 & 0.766 \\
\hline \multicolumn{15}{|l|}{$A x D$} \\
\hline Frontal & $-3,390$ & 0.041 & 360 & 0.840 & $-40,200$ & 0.088 & 17,900 & 0.757 & $-2,660$ & 0.207 & -993 & 0.547 & 59.9 & 0.986 \\
\hline Occipital & $-6,230$ & 0.149 & 4,250 & 0.216 & $-70,600$ & 0.098 & 95,500 & 0.423 & $-4,450$ & 0.236 & $-1,980$ & 0.500 & $-6,360$ & 0.298 \\
\hline Parietal & $-2,220$ & 0.081 & $-1,360$ & 0.422 & $-26,600$ & 0.241 & 14,500 & 0.789 & $-4,240$ & 0.036 & $-1,130$ & 0.496 & 5,740 & 0.087 \\
\hline Temporal & $-3,780$ & 0.564 & 4,630 & 0.474 & $-150,000$ & 0.020 & 1,880 & 0.992 & $-4,950$ & 0.496 & 2,130 & 0.705 & $-3,960$ & 0.738 \\
\hline \multicolumn{15}{|l|}{$F A$} \\
\hline Frontal & 22.8 & 0.001 & -0.51 & 0.961 & 145 & 0.154 & -466 & 0.044 & 25.6 & 0.030 & 4.95 & 0.614 & 19.0 & 0.354 \\
\hline Occipital & 12.0 & 0.034 & 7.75 & 0.160 & 89.9 & 0.123 & -351 & 0.005 & 7.86 & 0.199 & -2.26 & 0.640 & 10.6 & 0.287 \\
\hline Parietal & 15.7 & 0.035 & -10.4 & 0.359 & 141 & 0.183 & -351 & 0.176 & 25.06 & 0.041 & 5.43 & 0.590 & 1.13 & 0.958 \\
\hline Temporal & 26.8 & 0.003 & 3.61 & 0.748 & 148 & 0.152 & -348 & 0.147 & 13.1 & 0.238 & -5.66 & 0.515 & -12.1 & 0.510 \\
\hline
\end{tabular}

MMSE = Mini-Mental State Examination; Trail = Trail Making Test B; Span forward = Wechsler Memory Scale-III Longest span forward; Span backward = Wechsler Memory Scale-III Longest span backward. $\beta$ represents the nonstandardized regression coefficient.

Table 4. Regression coefficients of the correlation between white matter tract FA, MD, RD and AD with cognitive test scores among participants with $\mathrm{AD}$

\begin{tabular}{|c|c|c|c|c|c|c|c|c|c|c|c|c|c|c|}
\hline & \multicolumn{2}{|c|}{ MoCA $(n=14)$} & \multicolumn{2}{|c|}{ MMSE $(n=10)$} & \multicolumn{2}{|c|}{ Stroop $(n=9)$} & \multicolumn{2}{|c|}{ Trail $(\mathrm{n}=8)$} & \multicolumn{2}{|c|}{$\begin{array}{l}\text { Span forward } \\
(\mathrm{n}=10)\end{array}$} & \multicolumn{2}{|c|}{$\begin{array}{l}\text { Span } \\
\text { backward } \\
(\mathrm{n}=10)\end{array}$} & \multicolumn{2}{|c|}{$\begin{array}{l}\text { Letter } \\
\text { Number } \\
\text { Sequencing } \\
(\mathrm{n}=9)\end{array}$} \\
\hline & $\beta$ & $\mathrm{p}$ & $\beta$ & $\mathrm{p}$ & $\beta$ & $\mathrm{p}$ & $\beta$ & $\mathrm{p}$ & $\beta$ & $\mathrm{p}$ & $\beta$ & $\mathrm{p}$ & $\beta$ & $\mathrm{p}$ \\
\hline \multicolumn{15}{|l|}{$M D$} \\
\hline Frontal & $-5,260$ & 0.287 & $-2,380$ & 0.345 & 8,960 & 0.854 & $-307,000$ & 0.353 & 2,270 & 0.548 & 5,030 & 0.290 & $-5,620$ & 0.589 \\
\hline Occipital & $-10,920$ & 0.080 & -497 & 0.893 & 8,470 & 0.950 & 118,000 & 0.909 & -350 & 0.973 & 7,460 & 0.577 & 12,500 & 0.668 \\
\hline Parietal & 1,060 & 0.651 & -30.4 & 0.980 & -176 & 0.994 & $-92,900$ & 0.563 & 360 & 0.825 & 2,030 & 0.317 & 3,920 & 0.401 \\
\hline Temporal & $-12,000$ & 0.031 & $-3,610$ & 0.255 & 101,000 & 0.130 & $-573,000$ & 0.280 & 6,490 & 0.243 & 8,300 & 0.252 & 7,130 & 0.661 \\
\hline \multicolumn{15}{|l|}{$R D$} \\
\hline Frontal & $-5,220$ & 0.303 & $-2,300$ & 0.375 & 8,850 & 0.859 & $-329,000$ & 0.326 & 2,160 & 0.575 & 5,140 & 0.290 & $-5,660$ & 0.593 \\
\hline Occipital & $-10,500$ & 0.061 & 170 & 0.961 & 51,000 & 0.659 & 19,600 & 0.982 & $-4,230$ & 0.641 & 1,160 & 0.923 & 2,570 & 0.919 \\
\hline Parietal & 1,180 & 0.626 & -67.9 & 0.957 & -481 & 0.984 & $-94,200$ & 0.573 & 336 & 0.843 & 2,060 & 0.331 & 3,990 & 0.415 \\
\hline Temporal & $-11,400$ & 0.031 & $-3,500$ & 0.244 & 95,400 & 0.140 & $-599,000$ & 0.223 & 5,540 & 0.305 & 7,460 & 0.287 & 6,200 & 0.691 \\
\hline \multicolumn{15}{|l|}{$A x D$} \\
\hline Frontal & $-5,020$ & 0.275 & $-2,490$ & 0.285 & 7,050 & 0.878 & $-264,000$ & 0.403 & 2,470 & 0.486 & 4,870 & 0.277 & $-5,300$ & 0.590 \\
\hline Occipital & $-8,770$ & 0.205 & $-2,100$ & 0.568 & $-118,000$ & 0.357 & 37,000 & 0.970 & 7,730 & 0.419 & 17,100 & 0.141 & 26,900 & 0.331 \\
\hline Parietal & 429 & 0.833 & 93.0 & 0.929 & 3,770 & 0.846 & $-92,000$ & 0.528 & 626 & 0.653 & 1,900 & 0.273 & 4,210 & 0.285 \\
\hline Temporal & $-12,070$ & 0.057 & $-3,920$ & 0.259 & 92,900 & 0.230 & $-531,000$ & 0.383 & 9,240 & 0.115 & 12,310 & 0.105 & 10,000 & 0.561 \\
\hline \multicolumn{15}{|l|}{$F A$} \\
\hline Frontal & 16.5 & 0.667 & -9.33 & 0.645 & -83.8 & 0.810 & 2,890 & 0.199 & 17.8 & 0.512 & -4.12 & 0.909 & 64.6 & 0.373 \\
\hline Occipital & 19.1 & 0.174 & -9.19 & 0.277 & -167 & 0.271 & 234 & 0.847 & 12.7 & 0.289 & 12.1 & 0.447 & 22.0 & 0.522 \\
\hline Parietal & -21.4 & 0.432 & -0.61 & 0.965 & -25.9 & 0.937 & 652 & 0.782 & 11.8 & 0.583 & 1.62 & 0.954 & -12.1 & 0.864 \\
\hline Temporal & 38.3 & 0.020 & 6.88 & 0.493 & -257 & 0.164 & 1,440 & 0.292 & 8.61 & 0.589 & 10.3 & 0.618 & 16.0 & 0.716 \\
\hline
\end{tabular}

MMSE = Mini-Mental State Examination; Trail = Trail Making Test B; Span forward = Wechsler Memory Scale-III Longest span forward; Span backward = Wechsler Memory Scale-III Longest span backward. $\beta$ represents the nonstandardized regression coefficient. 
was associated with increased MD/RD in temporal SWM. Decreased processing speed assessed by the Trail test was associated with increased RD/decreased FA in occipital SWM. There were no associations between SWM and cognitive function in participants with AD.

A limitation of this study is the small sample size. Cognitive test scores were only available in a subset of participants leading to smaller sample sizes for cognitive correlations. The small sample size may have limited the ability to detect diffusion differences between participants with $\mathrm{AD}$ and controls or detect cognitive associations with SWM. Alternatively, the disruption of SWM in AD may have contributed to the lack of cognitive associations for SWM in AD. Additional limitations of this study are the multiple comparisons, which may have led to falsepositive results, and the accuracy of tractography. The complex architecture of SWM makes it difficult to follow the course of tracts through crossing or abutting fibers [28]. Although some tracts may not have been tracked at all or not correctly, this study and earlier ones have demonstrated that it is possible to perform tractography for SWM $[7,28]$.

With tractography, we were able to reconstruct the extensive cortico-cortical connections of SWM. This network of connections between cortical regions assumes an important role in cognition. At the level of cerebral lobes, this study demonstrated that SWM has regionspecific roles in normal cognition. In healthy persons, tractography of SWM can be used to characterize in more detail the relationship between regional cortical connectivity and specific cognitive processes. In AD, tractography of temporal SWM may represent a novel biomarker of neuronal injury that may aid in diagnosis and disease monitoring.

\section{Statement of Ethics}

All procedures followed were in accordance with the ethical standards of the responsible committee on human experimentation (institutional and national) and with the Helsinki Declaration of 1975, as revised in 2000. Informed consent was obtained from all patients for being included in the study.

\section{Disclosure Statement}

All authors declare that they have no conflicts of interest.

\section{References}

$>1$ Amlien IK, Fjell A: Diffusion tensor imaging of white matter degeneration in Alzheimer's disease and mild cognitive impairment. Neuroscience 2014;12:206-215.

-2 Bartzokis G: Alzheimer's disease as homeostatic responses to age-related myelin breakdown. Neurobiol Aging 2011;32:1341-1371.

-3 Fornari E, Maeder P, Meuli R, Ghika J, Knyazeva MG: Demyelination of superficial white matter in early Alzheimer's disease: a magnetization transfer imaging study. Neurobiol Aging 2012;33:428.e7-e19.

$>4$ Reveley C, Seth AK, Pierpaoli C, Silva AC, Yu D, Saunders RC, Leopold DA, Ye FQ: Superficial white matter fiber systems impede detection of long-range cortical connections in diffusion MR tractography. Proc Natl Acad Sci USA 2015;26:E2820-E2828.

5 Phillips OR, Joshi SH, Piras F, Orfei MD, Iorio M, Narr KL, Shattuck DW, Caltagirone C, Spalletta G, Di Paola M: The superficial white matter in Alzheimer's disease. Hum Brain Mapp 2016;37:1321-1334.

6 Le Bihan D: Looking into the functional architecture of the brain with diffusion MRI. Nat Rev Neurosci 2003; 4:469-480.

7 Nazeri A, Chakravarty MM, Rajji TK, Felsky D, Rotenberg DJ, Mason M, Xu LN, Lobaugh NJ, Mulsant BH, Voineskos AN: Superficial white matter as a novel substrate of age-related cognitive decline. Neurobiol Aging 2015;36:2094-2106. 
8 Acosta-Cabronero J, Williams GB, Pengas G, Nestor PJ: Absolute diffusivities define the landscape of white matter degeneration in Alzheimer's disease. Brain 2010;133:529-539.

-9 Agosta F, Pievani M, Sala S, Geroldi C, Galluzzi S, Frisoni GB, Filippi M: White matter damage in Alzheimer disease and its relationship to gray matter atrophy. Radiology 2011;258:853-863.

-10 Bosch B, Arenaza-Urquijo EM, Rami L, Sala-Llonch R, Junqué C, Solé-Padullés C, Peña-Gómez C, Bargalló N, Molinuevo JL, Bartrés-Faz D: Multiple DTI index analysis in normal aging, amnestic MCI and AD. Relationship with neuropsychological performance. Neurobiol Aging 2012;33:61-74.

11 Damoiseaux JS, Smith SM, Witter MP, Sanz-Arigita EJ, Barkhof F, Scheltens P, Stam CJ, Zarei M, Rombouts SA: White matter tract integrity in aging and Alzheimer's disease. Hum Brain Mapp 2009;30:1051-1059.

-12 Salat DH, Tuch DS, van der Kouwe AJ, Greve DN, Pappu V, Lee SY, Hevelone ND, Zaleta AK, Growdon JH, Corkin S, Fischl B, Rosas HD: White matter pathology isolates the hippocampal formation in Alzheimer's disease. Neurobiol Aging 2010;31:244-256.

-13 Sexton CE, Kalu UG, Filippini N, Mackay CE, Ebmeier KP: A meta-analysis of diffusion tensor imaging in mild cognitive impairment and Alzheimer's disease. Neurobiol Aging 2011;32:2322.e5-e18.

14 Shu N, Wang Z, Qi Z, Li K, He Y: Multiple diffusion indices reveals white matter degeneration in Alzheimer's disease and mild cognitive impairment: a tract-based spatial statistics study. J Alzheimers Dis 2011;3:275285.

15 Nasreddine ZS, Phillips NA, Bédirian V, Charbonneau S, Whitehead V, Collin I, Cummings JL, Chertkow H: The Montreal Cognitive Assessment, MoCA: a brief screening tool for mild cognitive impairment. J Am Geriatr Soc 2005;53:695-699.

16 Folstein MF, Folstein SE, McHugh PR: 'Mini-mental state.' A practical method for grading the cognitive state of patients for the clinician. J Psychiatr Res 1975;12:189-198.

17 Stroop JR: Studies of interference in serial verbal reactions. J Exp Psychol 1935;18:643-662.

18 Corrigan JD, Hinkeldey MS: Relationships between parts A and B of the Trail Making Test. J Clin Psychol 1987; 43:402-409.

19 Wechsler D: Wechsler Memory Scale, ed 3. San Antonio, The Psychological Corporation, 1997.

20 McKhann GM, Knopman DS, Chertkow H, Hyman BT, Jack CR Jr, Kawas CH, Klunk WE, Koroshetz WJ, Manly JJ, Mayeux R, Mohs RC, Morris JC, Rossor MN, Scheltens P, Carrillo MC, Thies B, Weintraub S, Phelps CH: The diagnosis of dementia due to Alzheimer's disease: recommendations from the National Institute on AgingAlzheimer's Association workgroups on diagnostic guidelines for Alzheimer's disease. Alzheimers Dement 2011;7:263-269.

-21 Reginold W, Itorralba J, Tam A, Luedke AC, Fernandez-Ruiz J, Reginold J, Islam O, Garcia A: Correlating quantitative tractography at 3T MRI and cognitive tests in healthy older adults. Brain Imaging Behav 2015, Epub ahead of print.

-22 Reginold W, Itorralba J, Tam A, Luedke AC, Fernandez-Ruiz J, Reginold J, Islam O, Garcia A: Tractography at 3T MRI of corpus callosum tracts crossing white matter hyperintensities. AJNR Am J Neuroradiol 2016, Epub ahead of print.

-23 Reginold W, Luedke AC, Tam A, Itorralba J, Fernandez-Ruiz J, Reginold J, Islam O, Garcia A: Cognitive function and 3-tesla magnetic resonance imaging tractography of white matter hyperintensities in elderly persons. Dement Geriatr Cogn Disord Extra 2015;5:387-394.

-24 Smith SM, Jenkinson M, Woolrich MW, Beckmann CF, Behrens TE, Johansen-Berg H, Bannister PR, De Luca M, Drobnjak I, Flitney DE, Niazy RK, Saunders J, Vickers J, Zhang Y, De Stefano N, Brady JM, Matthews PM: Advances in functional and structural MR image analysis and implementation as FSL. Neuroimage 2010; 23:S208-S219.

-25 Bokde AL, Teipel SJ, Schwarz R, Leinsinger G, Buerger K, Moeller T, Möller HJ, Hampel H: Reliable manual segmentation of the frontal, parietal, temporal, and occipital lobes on magnetic resonance images of healthy subjects. Brain Res Brain Res Protoc 2005;14:135-145.

-26 Pini L, Pievani M, Bocchetta M, Altomare D, Bosco P, Cavedo E, Galluzzi S, Marizzoni M, Frisoni GB: Brain atrophy in Alzheimer's disease and aging. Ageing Res Rev 2016, Epub ahead of print.

27 Acosta-Cabronero J, Alley S, Williams GB, Pengas G, Nestor PJ: Diffusion tensor metrics as biomarkers in Alzheimer's disease. PLoS One 2012;7:e49072.

28 Oishi K, Zilles K, Amunts K, Faria A, Jiang H, Li X, Akhter K, Hua K, Woods R, Toga AW, Pike GB, Rosa-Neto P, Evans A, Zhang J, Huang H, Miller MI, van Zijl PC, Mazziotta J, Mori S: Human brain white matter atlas: identification and assignment of common anatomical structures in superficial white matter. Neuroimage 2008;15: 447-457. 